\title{
Graft Intolerance Syndrome in Children Treated with Kidney Embolization: A Case Report
}

\section{Reyner Loza1, Nathalie Rodríguez¹, Angelica Ynguil'1, Fernando Arias' ${ }^{1}$, Gustavo Araujo², Luis Zegarra ${ }^{3}$}

\author{
${ }^{1}$ Department of Pediatrics, Pediatric Kidney Transplant Unit, Cayetano Heredia National Hospital, Lima, Peru \\ ${ }^{2}$ Department of Radiology Center for Radiological and Endoscopic Interventions (REI), Lima, Peru \\ ${ }^{3}$ Department of Surgery Renal Transplant Unit, Cayetano Heredia Hospital, Lima, Peru \\ Email: reyner.loza@upch.pe
}

How to cite this paper: Loza, R., Rodríguez, N., Ynguil, A., Arias, F., Araujo, G. and Zegarra, L. (2020) Graft Intolerance Syndrome in Children Treated with Kidney Embolization: A Case Report. Open Journal of Nephrology, 10, 361-366.

https://doi.org/10.4236/ojneph.2020.104035

Received: October 13, 2020

Accepted: December 12, 2020

Published: December 15, 2020

Copyright (อ 2020 by author(s) and Scientific Research Publishing Inc. This work is licensed under the Creative Commons Attribution International License (CC BY 4.0).

http://creativecommons.org/licenses/by/4.0/

\begin{abstract}
During post-transplant evolution, adolescents may present problems with adherence to treatment, becoming a high-risk group for graft loss. Here, we report a case which describes an adolescent patient who lost a graft due to humoral rejection associated with lack of adherence to treatment. During chronic peritoneal dialysis therapy, the patient developed pain and increased volume in the graft area, fever, gross hematuria and leukocyturia upon urine examination. The patient was diagnosed with graft immune intolerance syndrome and transplantectomy was suggested. Finally, a graft embolization was performed. A decrease in symptoms was observed until the patient became asymptomatic.
\end{abstract}

\section{Keywords}

Kidney Transplantation, Children, Embolization Therapeutic, Graft Rejection

\section{Introduction}

Renal transplantation is the ideal treatment for adolescents with chronic kidney failure. However, during the post-transplant evolution, adolescents may present problems with adherence to treatment, becoming a high-risk group for graft loss.

Graft intolerance is a syndrome characterized by enlargement of the graft, hematuria, signs of acute inflammation, anemia with resistance to erythropoietin, fever, malaise, raised C-reactive protein. This reflects the chronic inflamma- 
tion that is induced by graft failure and occurs in the first year of dialysis in $30 \%$ to $50 \%$ of patients who have lost the graft.

But there are no cases reported in children with this syndrome and with surgical treatment of embolization. As previously described, we believe that it is important to report this case about a girl who lost the graft.

\section{Case Report}

16-year-old female adolescent transplanted at 9 years of age from an unrelated living donor. She received triple maintenance therapy with steroids, mycophenolate mofetil and tacrolimus, and the etiology of the primary kidney disease was renal hypoplasia. Seven years after the transplant, she lost the graft due to two episodes of humoral rejection due to lack of adherence to treatment. She returned to the chronic peritoneal dialysis program, tacrolimus was suspended, the dose of prednisone was reduced to $7.5 \mathrm{mg}$ per day, mycophenolate mofetil was reduced to $250 \mathrm{mg}$ daily.

During evolution, she presented episodes of fever, chills, weakness, pain in the graft area, gross hematuria, and refractory anemia in response to treatment, despite high doses of erythropoietin.

Physical examination documented high blood pressure, tachycardia, pale skin, cervical and inguinal lymphadenopathy, palpable mass in the iliac fossa and in the right flank measuring $19 \times 17 \mathrm{~cm}$, which was determined to be a tumor mass, ruling out renal abscess and tuberculosis (TBC). Wide spectrum antibiotic was initiated, urine analysis showed hematuria with leukocyturia. Cultures were negative for common germs including for TBC, as well as PCR studies for cytomegalovirus and Epstein-Barr virus. The anemia study included bone marrow aspirate, showing hypo regeneration of the erythroid series, while a biopsy of the lymph node showed a non-specific inflammatory reaction.

Renal ultrasound of the $126 \mathrm{~mm} \times 63 \mathrm{~mm}$ graft produced a solid, hyperechogenic image with well-defined contours. Using computed tomography (CT) of the kidney, a hyper vascularized renal graft was observed, enlarged and with hypo-uptake area compatible with necrosis (Figure 1).

With all this information, the diagnosis of graft intolerance syndrome was raised, restarting immunosuppressive therapy with mycophenolate mofetil 500 $\mathrm{mg} /$ day and low-dose corticosteroids $7.5 \mathrm{mg} /$ day, surgical removal was considered. Given the morbidity related to transplantectomy, the embolization of the graft was performed by an interventional radiologist over three sessions the kidney is reached via the femoral artery and the embolization of the distal vessels begins with 500-micron Embozene microspheres mixed with contrast until stasis of the intrarenal arteries is achieved, followed by fragments of Gelfoam ${ }^{\triangleright}$ until the renal artery is occluded, at the end of which the graft was decreased in size and a decrease in vascularization was observed in imaging studies (Figures 2-4). In the color Doppler, little blood flow was observed. Likewise, the patient's fever and general discomfort diminished in conjunction with a decrease in the size of the 


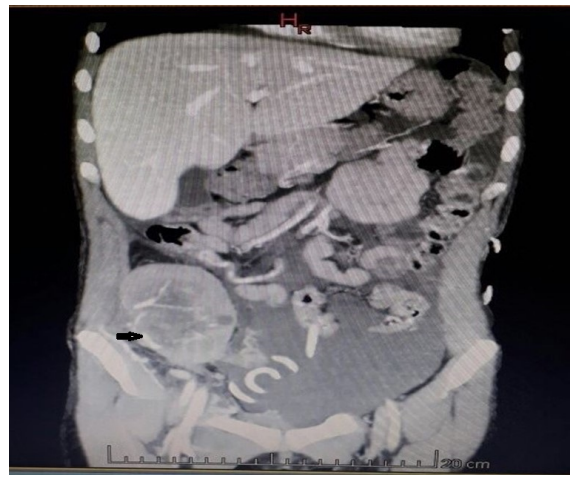

Figure 1. Highly vascularized kidney graft enlarged with hypo-uptake area compatible with necrosis was observed.

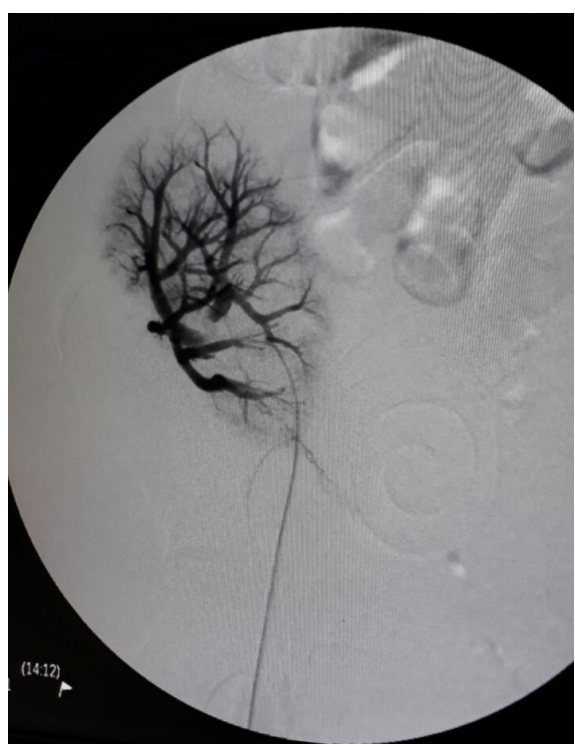

Figure 2. Digital subtraction image: graft arteries.

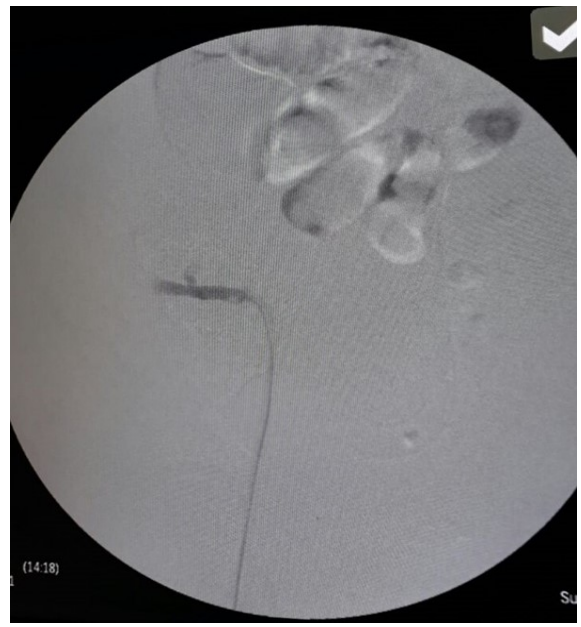

Figure 3. Complete embolization of the renal artery. 


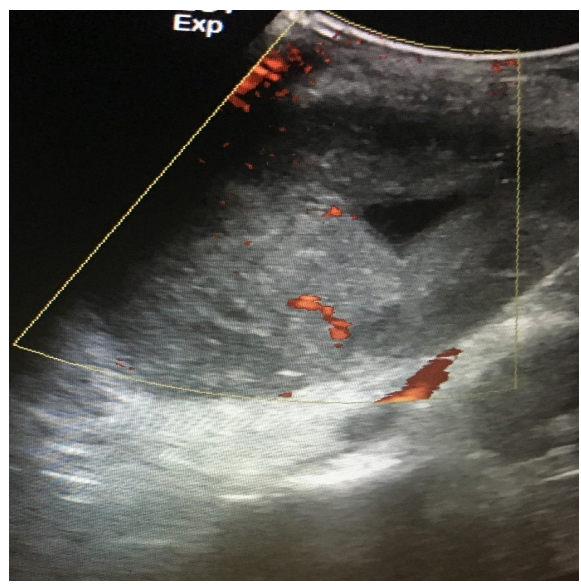

Figure 4. Decreased blood flow was observed by doppler.

graft, as well as an improvement in laboratory inflammation indicators and favorable clinical evolution. Antibiotic prophylaxis was used.

\section{Discussion}

Immune graft intolerance syndrome is a subclinical state of chronic inflammation generated by a non-functioning graft. It is characterized by fever of non-infectious origin, hematuria, pain and/or an increase in the size of the graft [1].

After a failed transplant, managing a non-functioning graft with pain or recurring infections is always a problem. Graft nephrectomy is recommended in case of failure in the period of 3 to 6 months as part of the current observation practice after transplantation.

The conventional treatment for this syndrome has been a surgical allograft nephrectomy. However, this procedure is associated with a substantial rate of potentially serious complications, such as vascular injury, bleeding, bruising, abscess, wound infection, and even mortality [2] [3]. With the development and improvement of interventional Angio radiology techniques, we have a new therapeutic weapon for the management of these cases: percutaneous intra-arterial embolization of the graft, which is a minimally invasive alternative to surgical excision, since it reduces complications [4] [5].

A common indication for the elimination of the effects of the graft is the control of graft intolerance syndrome through embolization and restart immunosuppression treatment, as was the case in our patient. The most frequent complication after this procedure is post-embolization syndrome, with an incidence of $68 \%$, characterized by fever lasting 2 to 5 days in $59 \%$ of cases, local pain, hematuria, nausea and vomiting, which, if persisting, requires the presence of vascularization of the graft and the persistence of immune intolerance to be ruled out [6].

Moreover, it is important to bear in mind that if embolization attempts fail, 
$20 \%$ of patients will need a nephrectomy.

However, percutaneous embolization was associated with lower mortality and morbidity rates. Embolization is an attractive technique that can be considered in the treatment of kidney grafts that do not work [7] [8].

Other indications for kidney embolization in pediatric nephrology are arterial aneurysms and arteriovenous malformations, functional exclusion and polycystic kidney disease before transplantation, angiomyolipoma (mainly in the context of tuberous sclerosis), and autosomal dominant polycystic kidney disease (ADPKD) before kidney transplantation [9] [10].

In the case of our patient, after three percutaneous embolizations, the patient evolved without any complication and comorbidity. The importance of this case is that this procedure has not been reported in post-transplant children [11].

\section{Conclusion}

Embolization is an attractive technique with low morbidity and mortality, which can be considered in the treatment of graft intolerance syndrome in post-transplant children.

\section{Consent}

Written informed consent was obtained from the patient for publication of this case report and the accompanying images.

\section{Conflicts of Interest}

The authors declare no conflicts of interest regarding the publication of this paper.

\section{References}

[1] Pérez-Flores, I., et al. (2009) Management of Failed Kidney Graft. Nephrectomy versus Embolization. Nefrología, 29, 54-61.

[2] O’Sullivan, D.C., Murphy, D.M., McLean, P. and Donovan, M.G. (1994) Transplant Nephrectomy over 20 Years: Factors Involved in Associated Morbidity and Mortality. Journal of Urology, 151, 855-858. https://doi.org/10.1016/S0022-5347(17)35105-4

[3] Voesten, H.G., Slooff, M.J., Hooykaas, J.A., Tegzess, A.M. and Kootstra, G. (1982) Safe Removal of Failed Transplanted Kidneys. British Journal of Surgery, 69, 480-481.

[4] González-Satué, D., Riera, I., Franco, E., et al. (2000) Percutaneous Embolization of the Renal Transplant Graft as a Therapeutic Alternative to Surgical Transplant. $A c$ tas Urológicas Españolas, 24, 319-324. https://doi.org/10.1016/S0210-4806(00)72455-7

[5] González-Satué, C., Riera, L., Franco, E., Escalante, E., Dominguez, J. and Serrallach, N. (2000) Percutaneous Embolization of the Failed Renal Allograft in Patients with Graft Intolerance Syndrome. BJU International, 86, 610-612.

[6] Takase, H.M., Contti, M.M., Nga, H.S., et al. (2018) Nephrectomy versus Embolization of Non-Functioning Renal Graft: A Systematic Review with a Proportional 
Meta-Analysis. Annals of Transplantation, 23, 207-217.

https://doi.org/10.12659/AOT.907700

[7] Grenier, N., Petitpierre, F., Le Bras, Y., Lasserre, A.S. and Cornelis, F. (2016) Renal Embolization. Néphrologie \& Thérapeutique, 12, S139-S143.

https://doi.org/10.1016/j.nephro.2016.01.009

[8] Al Badaai, G., Pernin, V., Garrigue, V., et al. (2017) Renal Graft Intolerance Syndrome in Late Graft Failure Patients: Efficacy and Safety of Embolization as First-Line Treatment Compared to Surgical Removal. Transplant International, 30, 484-493.

[9] Yeast, C., Riley, J.M., Holyoak, J., Ross Jr., G., Weinstein, S. and Wakefield, M. (2016) Use of Preoperative Embolization Prior to Transplant Nephrectomy. International Brazilian Urology, 42, 107-112.

https://doi.org/10.1590/S1677-5538.IBJU.2015.0052

[10] Ye, W., Voss, M.D. and Athreya, S. (2018) Volume Reduction in Enlarged Kidneys in Autosomal Dominant Polycystic Kidney Disease (ADPKD) Prior to Renal Transplant with Transcatheter Arterial Embolization (TAE): A Systematic Review and Meta-Analysis. Cardio Vascular and Interventional Radiology, 41, 828-834. https://doi.org/10.1007/s00270-018-1890-7

[11] Bunthof, K.L.W., Verhoeks, C.M., van den Brand, J.A.J.G. and Hilbrands, L.B. (2018) Graft Intolerance Syndrome Requiring Graft Nephrectomy after Late Kidney Graft Failure: Can It Be Predicted? A Retrospective Cohort Study. Transplant International, 31, 220-229. 